\title{
A thirty-nine-year survival with the Starr-Edwards mitral valve prosthesis
}

\section{Trentanove anni di sopravvivenza con una protesi mitralica di Starr-Edwards}

\author{
Sabino Scardi, Bruno Pinamonti ${ }^{1}$, Michele Moretti, Gianfranco Sinagra ${ }^{1}$
}

\begin{abstract}
A thirty-nine-year survival with the StarrEdwards mitral valve prosthesis. S. Scardi, B. Pinamonti, M. Moretti, G. Sinagra.

We report a case of a 57 year-old woman with StarrEdwards model 6120 mitral valve replacement and KayShiley bioprosthetic tricuspid valve replacement in 1968 at Niguarda Hospital in Milan. The mitral caged-ball has proved its excellent durability and its good hemodynamic performance in many patients, even if subject to high ten-
\end{abstract}

\begin{abstract}
dency to thrombosis. In literature there is no evidence of durability of this prosthesis longer than 35 years. Our patient after 39 years from mitral valve replacement lives a happy and fulfilling life (NYHA II), with no evidence of hemolysis, ball variance, symptomatic embolization or major bleeding.

Keywords: Starr-Edwards prosthesis, mitral valve, valvular surgery.
\end{abstract}

Monaldi Arch Chest Dis 2008; 70: 34-37.

School of Cardiology, 1 Department of Cardiology, University Hospital Trieste, Italy.

Corresponding author: Prof Sabino Scardi; Via Moro, 5 - I-34136Trieste, Italy; E-mail sabino.scardi@libero.it

The Starr-Edwards caged-ball valve (SECBV) was first implanted on a man in September 21, 1960 [1]. A design breakthrough was achieved by Starr and Edwards, who engineered an integrated structure consisting of a stainless-steel cage, a fixation ring made from knitted Teflon cloth, and a heatcured Silastic ball. Design criteria were formulated. The materials had to be chemically inert, compatible with human tissue, atrumatic to blood, and nonthrombogenic. They also had to retain their structural properties over many years and lend themselves to being engineered into a valve that was acceptable to patients, opened and closed rapidly in response to changes in the pressure gradient, and resulted in limited obstruction to forward flow and minimal regurgitation in the closed position. Finally, it had to be technically feasible to implant the prosthesis securely in an appropriate physiologic position [2].

Hemodynamic performance - data from Marrow et al. [3] - provides satisfactory conditions at rest and exercise, although the projection of the mitral valve prosthesis into the left ventricular chamber can produce outflow obstruction. The main advantage of the SECBV is its proven durability. We present a case of mitral valve (SECBV) replacement survived 39 years.

\section{Case report}

A 57-year-old woman presented with rheumatic fever when she was 9 years old (1954) and she had recurrence when she was 12 and 15 years old. When she was 17 years old (1962) she suffered from heart failure. At 23 years (functional class IV NYHA) she underwent an operation of mitral valve replacement with Starr-Edwards model 6120 and Kay-Shiley bioprosthetic tricuspid valve replacement (08.18.1968), at the Niguarda Hospital De Gasperi Center of Milan. At 38 years she was operated of hysterectomy due to uterus cancer. At 39 years of age, she was reoperated for implantation of Carpentier $n^{\circ} 34$ bioprosthetic valve $(03.12 .1984)$ due to structural tricuspid valve deterioration. At 50 years old a pacemaker VVI for atrial fibrillation and atrioventricular block was implanted (02.04.1995). When she was 52 years old, a second structural tricuspid valve deterioration (stenosis) was found (03.09.1997); subsequently, at 59 years, coronarography was performed, showing normal coronary arteries, and then the bioprosthetic tricuspid valve was replaced with a Carpentier - Edwards $n^{\circ} 29$ prothesis (05.06.2004).

At follow-up (08.07. 2007) after 39 years of mitral valve replacement, the patient has a normal life, with NYHA functional class II; BNP is $52 \mathrm{ng} / \mathrm{ml}$, no haemolysis nor ball variance is present. The patient has good compliance with warfarin-therapy, obtaining a convenient level of anticoagulation; in our specialist anticoagulation clinic $76 \%$ time was spent within the INR target 3-4.5 without symptomatic embolism or major bleeding. On auscultation a loud opening click is present, followed by multiple diastolic clicks of variable intensity produced by the ball bouncing in the cage; a flow systolic murmur is also present in the aortic position, produced by turbulence in the left ventricular outflow tract; a less intense click occurs with the closing of the valve. 
Electrocardiogram shows atrial fibrillation and a pacemaker VVI correctly functioning. At chest $\mathrm{X}$-rays severe biatrial enlargement is present, and the support ring and the cage are well seen (fig. 1).

Two-dimensional and M-mode echocardiogram shows a ball-in-cage Starr-Edwards prosthesis in mitral position of normal appearance, with the cage strut echoes and the anterior echo of the ball which opens moving toward the cage in diastole and closes in systole. A severe left atrial enlargement is evident (fig. 2). Colour-Doppler examination at prosthetic level typically shows multiple peripheral diastolic flow jets coming laterally from the cage and the ball. Continuous-wave Doppler study at the level of prosthetic flow shows the typical curve compatible with a well functioning prosthesis, with a moderate early diastolic gradient, a mild mean gradient and a mildly decreased functional prosthetic area, within normal limits for this type of prosthesis (fig. 3) [4].

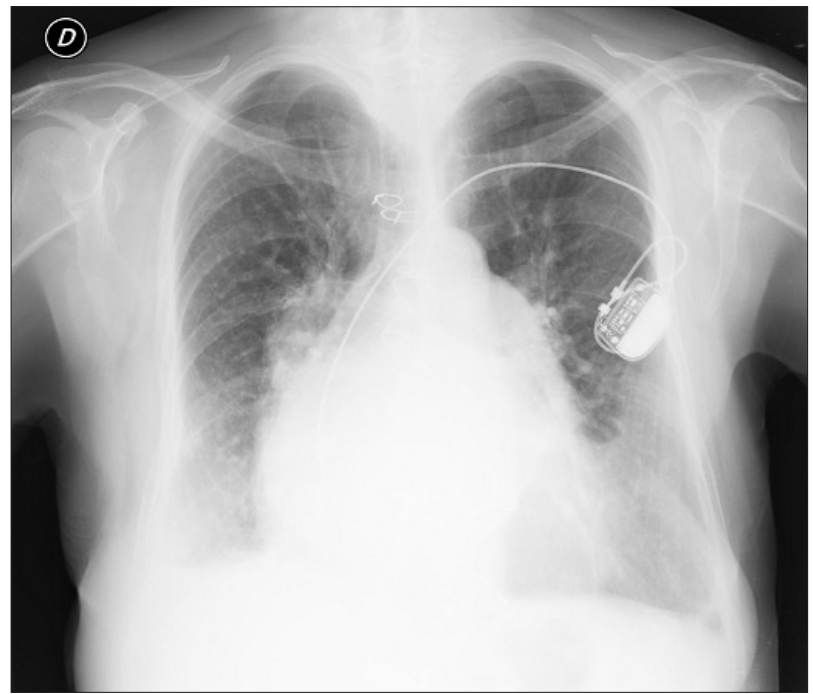

Figure 1. - Chest $\mathrm{x}$-ray of the patient showing severe cardiomegaly due to severe biatrial enlargement.

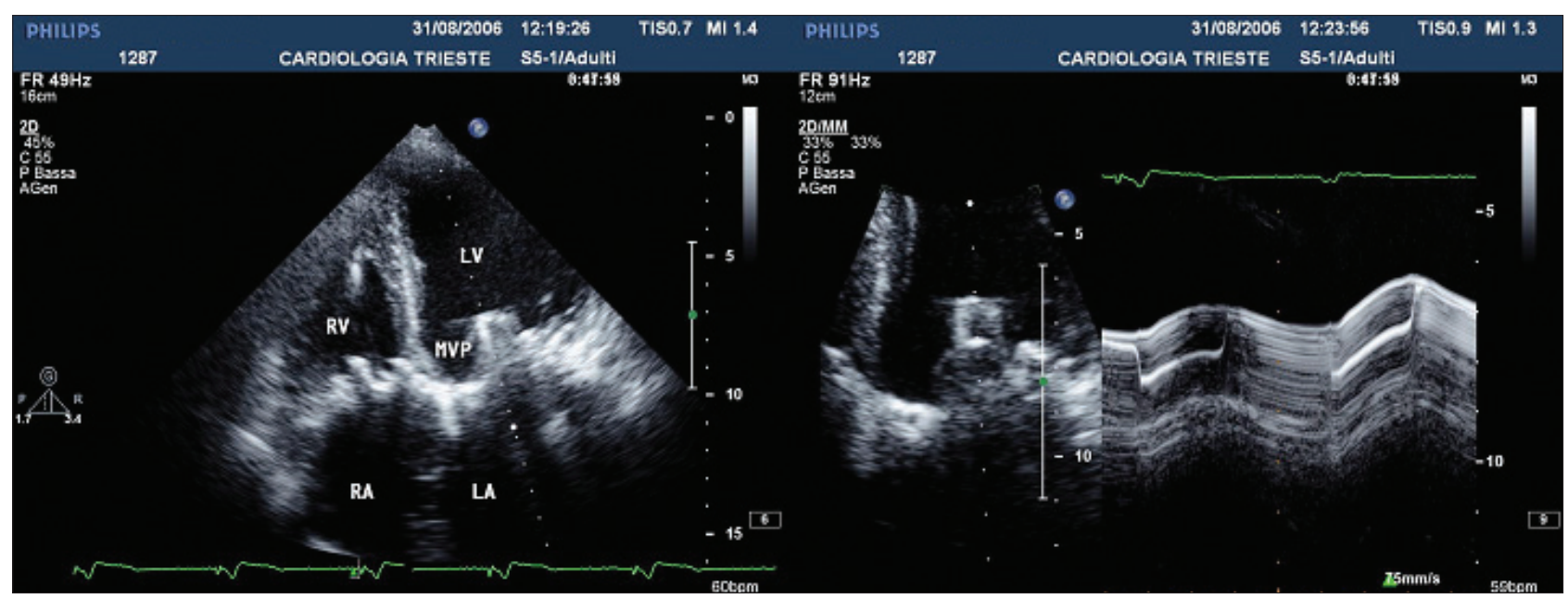

Figure 2A. - Two-dimensional echocardiographic examination from apical four-chamber view, systolic frame. The typical echocardiographic appearance of ball-in cage Starr-Edwards mitral prosthetis (MVP) is shown, with the ball in posterior closure position separated from the anterior echo of the cage. A severe left atrial (LA) enlargement is present. Other abbreviations: RV: right ventricle; LV: left ventricle, RA: right atrium.

Figure 2B. - M-mode echocardiographic examination from apical fourchamber view studying the Starr-Edwards mitral prosthesis, showing the diastolic contact between the echoes of the ball and of the cage, separating in systole.

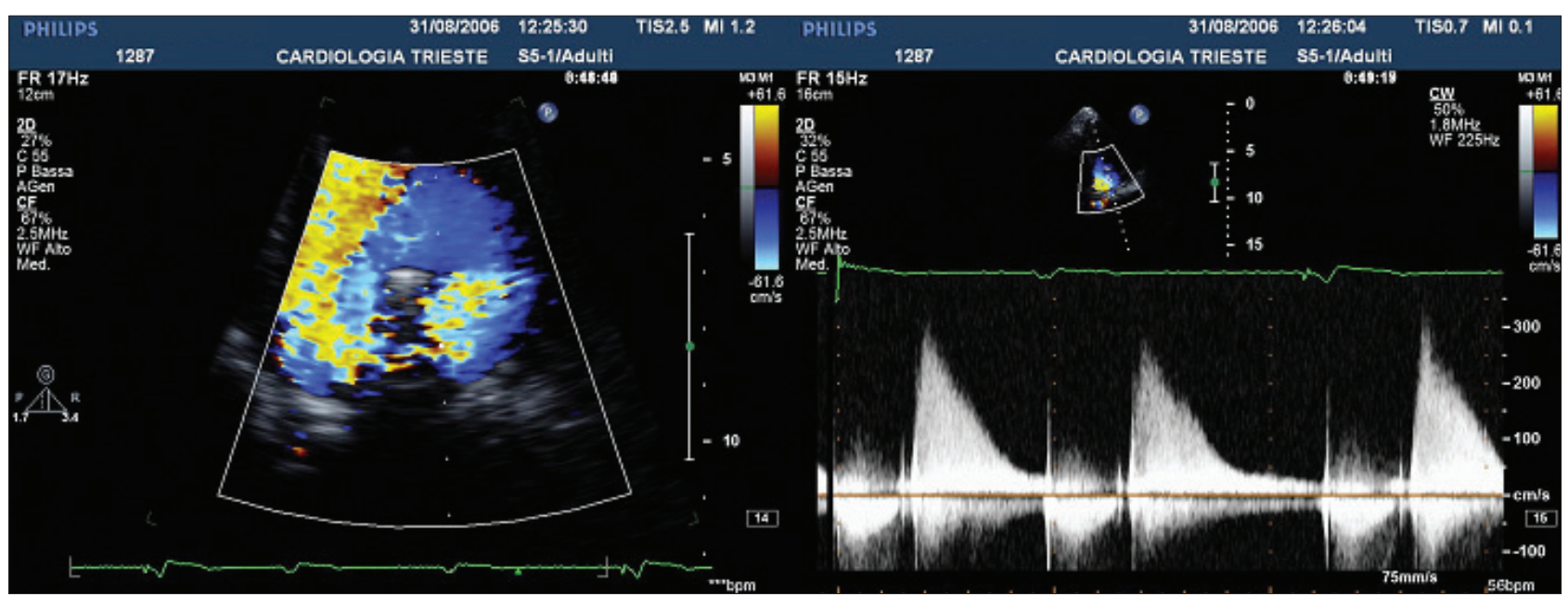

Figure 3A. - Colour-Doppler study at the level of mitral Starr-Edwards prosthesis, apical four chamber view, diastolic frame: multiple peripheral colour flow jets are seen.

Figure 3B. - Continuous wawe Doppler study at the level of Starr-Edwards prosthesis in mitral position, from apical approach, orienting the Doppler beam on one of the colour-flow jets: transprosthetic flow velocity corresponds to a moderate early diastolic gradient $(17 \mathrm{mmHg})$, a mild mean diastolic gradient $(8 \mathrm{mmHg})$ and a "functional" prosthetic area of 1.7 square $\mathrm{cm}$, within the normal level for this type of prosthesis. 


\section{Comment}

Long term survival of the individual patient is the primary goal of cardiac valve replacement. The SECBV has proved it exceptional longevity. The excellent record of valve performance in our patient is demonstrated by the fact that she survived 39 years after mitral valve replacement with SECBV, despite two subsequent reoperations for dysfunction of tricuspid valve bioprosthesis. In the literature there is no evidence of durability of this prosthesis longer than 35 years (5-6). Our patient after 39 years of mitral valve replacement lives a happy and fulfilling life (NYHA II), with no evidence of hemolysis, ball variance, symptomatic embolization or major bleeding.

Godje et al. [5] showed a survival rate at 10-2030 years of $75 \%, 36 \%$, and $23 \%$, respectively; freedom from all valve-related complications, reoperations, and valve related death was $73 \%, 35 \%$, and $14 \%$, respectively; notably $76 \%$ of the surviving patients was in NYHA class I and II at follow-up. Finally, De Santo et al. [6] recently confirmed the satisfactory durability of the SECBV over a long-period (27-year) with a retrospective study of 440 patients with isolated Starr-Edwards-mitral valve prostheses implanted after January 1974. The mortality at 30-day was $13 \%$. Mean follow-up was 5.157 years per patient, the mean observation time was $12 \pm 8$ years. Long-term mortality was $36 \%$; $64 \%$ of the deaths were cardiac (and 64\% of them were valve-related). Long-term survival at 1,5,10,15,20, and 25 years was $94 \%, 87 \%, 76 \%, 68 \%, 57 \%$, and $53 \%$, respectively. No primary mechanical failures was observed. The risk of complications and the survival rate of mitral prosthetic replacement depend on left atrial size, cardiac index, the presence of coronary heart disease and left ventricular ejection fraction.

The longevity of caged ball can be excellent. In fact, almost the only concern about its long-term performance relates to the possibility of alteration in the ball poppet itself, a change known as "ball variance ". The incidence of poppet degeneration was very low in mitral position.

In the registry of Godje et al. [5], the transvalvular gradient was between 9 and $30 \mathrm{mmHg} ; 68 \%$ showed no echocardiographic peculiarities and the left ventricular function was normal in $64 \%$. Thrombotic occlusion of the ball valve results from gradual stenosis of the orifice by pannus "ingrowth".

Patients with caged-ball valves carry a high risk of thromboembolism and require more intensive anticoagulation therapy (INR >4). In the Auckland study thromboembolic events occurred in $45 \%$ within 5 years, compared to $13 \%$ with bioprosthetic valves ( $\mathrm{p}=0-0001), 39 \%$ having long-term sequelae. The risk of late thromboembolism is approximately 4,5 events per 100 patients-year for patients that underwent operation from 1963 to 1971 [7].

In our patient prosthetic valve is well functioning after 39 years, as confirmed by echocardiographic data, with a moderate early diastolic gradient $(17 \mathrm{mmHg})$, a mild mean diastolic gradient $(8 \mathrm{mmHg})$ and a "functional" prosthetic area of 1.7 cmq. No symptomatic embolization or major he- morrhage were documented, thanks to an appropriate management of the anticoagulation therapy. The traditional management of oral anticoagulation is full of difficulties. Alternative models are therefore prospected (anticoagulant clinic, computerized software decision, portable coagulometer, self management). These new strategies can improve therapeutic control of oral anticoagulation [8].

In conclusion, the development of the StarrEdwards heart valve marked a new era in the treatment of valvular heart disease, and has dramatically altered the natural history of patients with valvular heart disease. The Starr-Edwards valve is rarely used today. At present, there is no single "ideal "valve that is appropriate for every single patient. Heart valve have come a long way, but we are proud to say that the Starr-Edwards valve's longevity is still the gold standard against which all others are compared [9]. Despite these glowing remarks the World Congress suggested us to recall the words of Paton and Vogel regarding the "perfect valve" that has yet to be realized. We may never have definitive results. At best, we may be able to say to patients. "Here and now, with this valve, at your age and with your ventricular function, you have certain percent chance of living 5 years, a different chance of living 10 years, and your chances of living without any complications are such and such. With your new valve you may have problems but we hope that the problems you have will be less serious that the problems you have had" [10]. In addressing these needs or otherwise meeting the challenge of sustained valve performance without thromboembolic risk, future solutions will probably combine more accurate computational tools that capture the complex environment of the prosthesis with molecularly engineered materials that have enhanced functionality and are assembled through microfabrication techniques. All of these developments will further the evolution of an advanced generation of heart-valve replacements whose form and function blur the distinction between the synthetic and the biologic [2].

\section{Riassunto}

Viene riferito il caso di una donna di 57 anni portatrice da 39 anni di una protesi mitralica di Starr-Edwards modello 6120 e di una bioprotesi tricuspidale di Kay-Shiley impiantate al Niguarda di Milano nel 1968. La protesi di Starr ha mostrato una eccellente durata e tuttora ha una buona funzione emodinamica. Infatti la paziente conduce una vita normale (NYHA II) e non ha avuto emolisi, embolie o emorragie sintomatiche o ball variance grazie anche al fatto che le è stata assicurata una eccellente continuità assistenziale. In letteratura non ci risulta una durata di questo tipo di protesi superiore a 35 anni.

Parole chiave: Protesi di Starr-Edwards, valvola mitralica, chirurgia valvolare.

Acknowledgements: Tank you to Dr Albert Starr, Eng. Lowell Edwards, Prof Renato Donatelli, nurse Gianna Benvenuto and Miss Sonia F. 


\section{References}

1. Starr A, Edwards ML. Mitral replacement: clinical experience with a ball-valve prosthesis. Ann Surg 1961; 154: 726.

2. Chaikof EL The development of prosthetic heart valveslesson in form and function. $N$ Engl J Med 2007; 357: 1368-1371.

3. Morrow AG, Oldham HN, Elkins RC, et al. Prosthetic replacement of the mitral valve: preoperative and postoperative clinical and hemodynamic assessment in 100 patients. Circulation 1967; 35: 962-979.

4. Oh JK, Steward JB, Tajik AJ, editors, The Echo Manual. 2nd edn, Philadelphia, Pennsylvania: Lippincott Williams \& Wilkins; 1999.

5. Godje OL, Fischlein T, Adelhard K, et al. Thirty-year results of Starr-Edwards prosthesis in the aortic and mitral position. Ann Thorac Surg 1997; 63: 613-619.
6. De Santo LS, Romano G, Petraio A, et al. Twenty-sevenyear follow up of the Starr-Edwards prosthesis implanted in the mitral position: an analysis of survival and valverelated complications. J Heart Valve Dis 2004; 1 (Supp 1): S21-S25.

7. North RA, Sadler L, Stewart K, et al. Long-term survival and valve-related complications in young women with cardiac valve replacement. Circulation 1999; 99: 26692676.

8. Tretjak I, Benvenuto G, Scardi S, et al. Control of anticoagulant therapy with portable prothrombin time device patients with mechanical heart valve prostheses: two-year follow-up. Monaldi Arch Chest Dis 2003; 60: 288-294.

9. Siposs GG. Memoirs of an early heart-valve engineer. Ann Thorac Surg 1989; 48: 56-57.

10. Paton BC, Vogel JHK. Introduction: valve replacement: the first quarter century. J Am Coll Cardiol 1985; 6: 897898. 